\title{
La référence aux personnages et aux lieux dans L'Occupation des sols
}

Michel Charolles et Benjamin Storme

\section{(2) OpenEdition \\ Journals}

Édition électronique

URL : http://journals.openedition.org/rsl/805

DOI : $10.4000 /$ rsl.805

ISSN : 2271-6246

Éditeur

Éditions Rue d'Ulm

Référence électronique

Michel Charolles et Benjamin Storme, "La référence aux personnages et aux lieux dans L'Occupation des sols », Revue Sciences/Lettres [En ligne], 3 | 2015, mis en ligne le 08 décembre 2014, consulté le 30 avril 2019. URL : http://journals.openedition.org/rsl/805; DOI : 10.4000/rsl.805

Ce document a été généré automatiquement le 30 avril 2019.

(c) Revue Sciences/Lettres 


\title{
La référence aux personnages et aux lieux dans L'Occupation des sols
}

\author{
Michel Charolles et Benjamin Storme
}

1 Les personnages occupent une place centrale dans le texte d'Echenoz qui se présente d'emblée comme un récit. Les sols, essentiellement urbains, ne leur disputent la prééminence que de façon épisodique, dans des passages à première vue incidents, où les bâtiments qui les occupent, les objets qui s'y trouvent, et d'autres notations sur lesquelles nous aurons l'occasion de revenir, prennent le dessus. Ces passages plus descriptifs rapportent aussi une histoire, celle des lieux, de leur conquête, et de leur transformation, au point que leur destin et celui des humains finissent, au fil du texte, par s'entremêler. Cette intrication est en réalité présente dès le début de la nouvelle, au travers du personnage de la mère qui est mentionnée tantôt en tant que personne, tantôt en tant qu'effigie représentée sur une façade d'immeuble.

2 Dans l'étude qui suit, nous allons examiner aussi précisément que possible, et au fur et à mesure de l'avancée du texte, comment sont introduits puis repris les personnages et les lieux. La première partie porte sur le paragraphe initial et traite à la fois des personnages et des lieux. Les deux parties suivantes sont consacrées aux expressions référentielles et anaphoriques renvoyant respectivement aux personnages et à l'espace (lieux et constructions les occupant).

\section{L'installation des personnages et des lieux dans I' incipit de L'Occupation des sols}

Comme tout avait brûlé - la mère, les meubles et les photographies de la mère -, pour Fabre et le fils Paul c'était tout de suite beaucoup d'ouvrage: toute cette cendre et ce deuil, déménager, courir se refaire dans les grandes surfaces. Fabre trouva trop vite quelque chose de moins vaste, deux pièces aux fonctions permutables sous une cheminée de brique dont l'ombre donnait l'heure, et qui avaient ceci de bien d'être assez proches du quai de Valmy. 

est en attente d'une principale à laquelle elle doit être rattachée. La construction est anticipante et le subordonnant comme indique la fonction que la subordonnée est prédestinée à remplir par rapport à la principale attendue. Le plus que parfait (avait brûlé) sélectionne une interprétation causale et exclut un lien de concomitance temporelle, qui serait possible avec l'imparfait (comme tout brûlait). La subordonnée fait allusion à un événement accompli qui est déclencheur. Le tour ne va pas sans rappeler l'incipit de Bouvard et Pécuchet (Comme il faisait une chaleur de trente-trois degrés, [...]), sauf que dans L'Occupation des sols, le plus que parfait renvoie à un événement révolu mais dont les effets se font ressentir jusqu'au commencement du récit.

4 Tout, pronom indéfini, réfère à un ensemble indéterminé d'entités spécifiques présumées accessibles dans le contexte. Dans les emplois en situation immédiate, comme Tout est à vendre... (par exemple dans un magasin d'antiquités), la situation d'énonciation et la prédication jouent un rôle essentiel dans la constitution de cet ensemble : tout = tout ce qui peut se vendre dans le magasin où la phrase est énoncée : en l'occurrence les meubles, les tableaux, les bibelots, ..., mais pas le comptoir derrière lequel est installé le marchand, ni les murs, le pas de porte, ... Tout exclut en principe les humains : dans Tout me plait dans ce club, tout = les locaux, les activités, ... à l'exclusion des humains, qui impliquerait tout le monde. Lorsque la prédication et la situation ne sont pas sélectives, comme dans tout fout le camp, tout commute avec rien qui n'est pas une expression référentielle : tout me plait ici et tout fout le camp seraient compris comme des énoncés thétiques (du genre de il pleut) exprimant un jugement global, non catégorique (i.e. dans lequel il n'y a pas attribution d'une propriété à une entité préalablement et indépendamment catégorisée). Dans l' incipit de L'Occupation des sols, le pronom indéfini tout ne peut être interprété que comme renvoyant à un ensemble indéterminé d'entités brûlables présumé accessible dans une certaine situation d'énonciation. Comme cette situation n'est pas spécifiée, l'occurrence de tout oblige à supposer un énonciateur prenant en charge l'assertion que 'tout avait brûlé', et qui donc a en tête une certaine situation à propos de laquelle il serait en droit de formuler un tel jugement. Quel peut être cet énonciateur? Par défaut, l'auteur omniscient, ou un narrateur ou encore un ou plusieurs participants au procès dont on s'attend à ce qu'il(s) soi(en)t introduit(s) dans la suite, ainsi que, le cas échéant, ce à quoi peut référer tout.

5 La suite répond partiellement à cette attente puisque le texte indique, dans une incise, ce qui a brûlé, à savoir : la mère, les meubles et les photographies de la mère. L'énumération est présentée comme exhaustive et, parmi les choses qui ont brûlé, se trouve notamment une personne évoquée à deux reprises et présentée comme étant la mère. Le groupe nominal défini présente cette personne comme satisfaisant au contenu prédicatif du nom mère qui est un nom relationnel. La description définie la mère est notoirement incomplète du fait que le nom relationnel n'est pas saturé par un nom propre (la mère de Un(e)tel(le)). Le groupe nominal défini singulier la mère présuppose l'existence d'une et une seule personne connue comme étant notoirement «la mère » dans le contexte, ce qui n'est possible que dans un micro univers de discours dans lequel, par connivence, on parle d'elle à l'aide de cette dénomination. Cette appellation n'implique pas nécessairement que le personnage en question soit la mère biologique d'au moins un des locuteurs au fait de cette convention de dénomination. Cette dénomination est partagée et, même si la mère en question est effectivement la mère d'un des locuteurs participant à l'univers du 
discours, elle est introduite en fonction de son rôle dans cet univers et non du lien qu'elle entretiendrait avec un de ses enfants, comme ce serait le cas si on avait eu ma/sa mère.

6 La vision est constative : la situation est rapportée de l'extérieur. Cette impression d'extériorité s'impose d'autant que le texte dit d'emblée que la mère (et pas seulement les photos d'elle) avait brûlé. Si en effet on peut brûler des humains (dans le sens de brûler une partie de leur peau) ou les faire brûler, il n'est par contre pas possible, linguistiquement, que des humains brûlent. Si des humains brûlent, il faut, pour la langue, qu'une force quelconque les aient faits brûler. Il n'est pas impossible de dire d'humains qu'ils ont brûlé, mais cela force à considérer qu'ils ont brûlé comme des choses, comme, en l'occurrence, les meubles et les photographies de la mère.

7 La suite : pour Fabre et le fils Paul c'était tout de suite beaucoup d'ouvrage relève du même registre constatif. Le récit n'avance pas : du fait de l'imparfait ${ }^{1}$, on en reste au relevé de la situation d'arrière-plan dans laquelle se trouvent les personnages. Le nom ouvrage est déverbal, il appelle un actant sujet en l'occurrence Fabre et le fils Paul pour qui l'incendie implique un travail. La construction est linguistiquement équivoque, le groupe prépositionnel pour Fabre et le fils Paul peut en effet être analysé soit comme un complément antéposé du nom ouvrage, soit comme un adverbial énonciatif de point de vue (proche de selon Fabre et le fils Paul). Les deux interprétations qui se confondent sémantiquement sont pratiquement indissociables.

8 L'antéposition de pour Fabre et le fils Paul satisfait d'emblée, au plan référentiel, à l'attente suscitée par l'allusion à la mère. Le mode d'introduction du fils est notoirement coopératif : le fait que Paul soit présenté comme le fils permet d'inférer avec assurance qu'il est bien le fils de la mère en question, ce que n'aurait pas permis le prénom seul, ni non plus, au moins univoquement, le possessif sans le nom propre (son fils) qui aurait pu être rattaché à Fabre. Le choix du défini le fils est toutefois relativement inattendu, on peut y voir un scrupule référentiel de l'auteur, mais la formulation connote, comme le défini la mère, un usage énonciativement marqué de type sociolectal. Le fait, par ailleurs, que Fabre soit introduit par son seul nom de famille le différencie de la mère et de son fils, ce qui laisse finalement ouverte l'éventualité qu'il ne soit ni le père ni le mari.

9 Il est possible que le pronom démonstratif dans c'était tout de suite beaucoup d'ouvrage soit compris, dans un premier temps, anaphoriquement comme renvoyant résomptivement au fait que "tout avait brûlé»), puis, rétroactivement comme faisant allusion cataphoriquement à cette cendre et ce deuil, déménager, courir se refaire... qui sont énumérés ensuite et qui explicitent les tâches auxquels ont dû faire face Fabre et le fils Paul. La cohésion du texte est maximale, l'interprétation cataphorique pousse le texte vers la suite mais, après que la subordonnée initiale a été rattachée à une principale, il manque toujours, comme on l'a déjà relevé, un événement d'avant-plan pour que le récit se mette pleinement en place.

Le fait que Fabre et Paul aient dû tout de suite déménager et courir se refaire est compris comme faisant allusion aux tâches (ouvrage) qu'ils ont dû effectuer. En revanche, l'allusion aux cendres et au deuil est plus surprenante. Les définis la cendre et le deuil auraient été possibles, sauf que les démonstratifs marquent une proximité psychologique avec les personnages (c'est comme cela qu'ils rendraient compte de la situation dans laquelle ils se sont retrouvés après l'incendie) et sollicitent l'expérience partagée des lecteurs sur le mode du "comme vous pouvez l'imaginer». Les définis, qui auraient exploité des connaissances encyclopédiques supposées communes, auraient été plus neutres. Avec les démonstratifs, la situation des personnages est envisagée à partir de la façon dont ils l'ont 
vécue et dont quiconque l'aurait vécue à leur place. Cet effet de proximité psychologique résulte de l'usage des démonstratifs dits mémoriels que l'on trouve dans les emplois du type La Grèce, tu te souviens, ces îles, ces plages... Dans les usages de ce type, les démonstratifs exploitent une connivence entre le locuteur et les interlocuteurs (ou forcent à penser qu'il en est ainsi), connivence qui serait beaucoup moins nette avec les îles, les plages...

11 La phrase qui suit et qui clôt l'incipit, Fabre trouva..., fixe le repère événementiel attendu. Le récit démarre sur le fond d'un état d'arrière-plan (alors) et il ne met en scène que le père, qui prend l'initiative réparatrice. La phrase apporte des informations descriptives sur le nouvel appartement, essentiellement sa taille et son emplacement, mais le compte rendu, loin d'être neutre, est émaillé d'indices d'évaluation:

- trop vite: trop adverbe de degré, associé à une polarité négative, implique la comparaison avec une norme implicite, en l'occurrence : «trop hâtivement par rapport à l'enjeu du choix »;

- quelque chose: groupe nominal indéfini référant à une entité spécifique qui n'est pas catégorisée au niveau de base attendu, ce qui peut suggérer que ce qu'a trouvé Fabre n'est pas clairement un appartement ;

- de moins vaste: comparativement au précédent logement (dont on ne saura rien d'autre jusqu'à la fin du récit) ;

- deux pièces, groupe nominal indéfini non référentiel (apposition descriptive), non aménagées (aux fonctions permutables) dans les combles (sous une cheminée de brique dont l'ombre donnait l'heure), deux espaces donc - un pour le père, un pour le fils?

- et qui avaient ceci de bien d'être assez proches du quai de Valmy: relative appositive qui vient corriger les aspects négatifs mentionnés dans la principale.

12 Les quelques indications fournies sur l'appartement trouvé par Fabre sont notoirement orientées, le mouvement concessif sur lequel s'achève l'incipit doit être pris en charge énonciativement. Les choix lexicaux, les marques d'appréciation négatives corrigées par la notation sur son emplacement, sont évidemment le fait de l'auteur, mais le lecteur tend à les attribuer aux personnages, en l'occurrence, pour les notations négatives, au fils, qui n'agit pas. Cette tendance s'impose par défaut : une fois entré dans la fiction, le lecteur ne peut s'empêcher de prêter aux protagonistes des états mentaux de toutes sortes, en particulier des émotions, des jugements de valeur, des intentions... expliquant leurs actions, leurs pensées et leurs propos. Ces inférences, fondées sur la psychologie du sens commun, multiplient les points de vue possibles et donc les énonciateurs à même de prendre en charge tout ou partie des énoncés dont les lecteurs prennent connaissance au fur et à mesure qu'ils avancent dans le texte. La part de ce qui revient à l'auteur s'en trouve réduite d'autant, au moins en apparence, car comme chacun sait - ou plutôt devrait savoir car tout l'exercice consiste à le faire oublier - c'est lui qui tire toutes les ficelles.

13 La machine mise en place par Echenoz dans le premier paragraphe de L'Occupation des sols est à cet égard d'une efficacité remarquable. Tout est fait pour pousser le lecteur en avant : subordonnée antéposée dont on attend la principale, surcharge de l'arrière-plan qui reporte l'attente d'un événement au passé simple, le tout débouchant sur une relative coordonnée qui, loin de conclure, comme elle en a l'air, introduit une énigme. Qu'est-ce qui, en effet, peut justifier que le fils Paul soit prêt à concéder à Fabre que les deux pièces sous les toits qu'il a dénichées, avaient ceci de bien d'être assez proches du quai de Valmy? Cette appréciation inattendue ne peut qu'intriguer et elle pousse, elle aussi, vers la suite 
du texte, tout en attirant discrètement l'attention sur l'espace urbain et la façon dont il peut être occupé...

\section{La référence aux personnages ${ }^{2}$}

\subsection{Fabre, Paul, et la mère}

Le soir après le dîner, Fabre parlait à Paul de sa mère, sa mère à lui Paul, parfois dès le dîner. Comme on ne possédait plus de représentation de Sylvie Fabre, il s'épuisait à vouloir la décrire toujours plus exactement : au milieu de la cuisine naquirent des hologrammes que dégonflait la moindre imprécision. Ça ne se rend pas, soupirait Fabre en posant une main sur sa tête, sur ses yeux, et le découragement l'endormait. Souvent ce fut à Paul de déplier le canapé convertible, transformant les choses en chambre à coucher.

Le SN défini temporel le soir renvoie génériquement à un ensemble de soirées et de dîners qui reste indéterminé depuis (on peut l'inférer) l'emménagement de Fabre (et du fils?) dans le nouvel appartement. Le groupe nominal détaché en tête de phrase et le groupe prépositionnel qui lui est adjoint (après le dîner) forment un constituant adverbial qui ouvre un cadre temporel compris comme habituel (comparer avec un soir après le dîner) dont la portée s'étend jusqu'à la fin du paragraphe. Ce cadre crée un nouveau contexte. Fabre, le fils et la mère sont redénommés : Fabre parlait à Paul de sa mère. Il lui parlait de sa mère aurait été possible, et même Il lui parlait d'elle (du fait de la marque de genre), mais l'effet aurait été différent, on serait resté dans la continuité des situations mentionnées dans l'incipit. Comme le détachement de l'adverbial temporel, les redénominations signalent qu'une page est déjà tournée dans l'histoire amorcée.

L'incise nominale, sa mère à lui Paul, est (anti)topicalisée, il y a reprise de sa mère, et focalisation sur Paul, ce qui implique une lecture contrastive et rectificative. La construction étant typique de l'oral, on a tendance à l'attribuer à Fabre et, donc à la lire comme un emprunt (non marqué) au discours qu'il adresse à son fils, comme s'il devait, à chacune de leur conversation, insister pour que celui-ci comprenne bien qu'il lui parlait de sa propre mère et non de la mère de quelqu'un d'autre. Pour le lecteur, il n'est pas exclu que l'adjonction de sa mère à lui Paul à la suite de Fabre parlait à Paul de sa mère l'amène, dans un premier temps, et assez paradoxalement, à se rendre compte de l'ambiguïté du possessif et à éliminer l'interprétation dans laquelle sa mère ferait allusion à la mère de Fabre, interprétation à laquelle il n'avait éventuellement pas songé. Il est vrai que l'ouverture d'un nouvel épisode temporel et les redénominations favorisent, comme on vient de le relever, une remise à zéro des compteurs référentiels, mais il n'est pas sûr que ces indications suffisent pour que les lecteurs, au contraire des protagonistes, oublient la mère qui avait brûlé... De sorte que l'incise, loin d'éliminer un rattachement référentiel indésirable, risque, au contraire, de les obliger à élaborer une interprétation qu'ils n'avaient pas envisagée.

16 L'emploi du nom propre complet dans la subordonnée causale détachée en tête de la phrase qui suit Comme on ne possédait plus de représentation de Sylvie Fabre ne s'imposait pas. Le personnage venant d'être focalisé (sans compter la différence de genre), un simple pronom de troisième personne aurait suffi. Le choix du nom propre s'explique par une autre raison, il permet d'apprendre, indirectement, que la mère est l'épouse de Fabre. Le pronom indéfini on pourrait être interprété déictiquement, par rapport à la situation rapportée, comme une sorte de nous, renvoyant à Fabre et à Paul. Mais le prédicat étant 
statique, la phrase est plutôt comprise comme exprimant un jugement thétique, sans sujet catégorique, sur le mode de il n'y avait plus de représentation de Sylvie. Dans les emplois de ce type, on ne réfère pas à un ou plusieurs actant(s) sujet(s) comme ce serait le cas si Echenoz avait écrit Comme ils ne possédaient plus de représentation de Sylvie Fabre. On est beaucoup plus subtil que ils car, même dans les emplois où il se contente de poser l'existence d'un événement, indépendamment des participants au procès, il manifeste toujours une sorte d'implication du locuteur ou du rédacteur dans celui-ci. Pour s'en rendre compte il suffit de penser aux emplois hypocoristiques en situation immédiate comme Alors on n'a plus faim (à un bébé). Dans les emplois de cette sorte : tu serais neutre, il trop distancé (cf. la non-personne chère à Benveniste), quant à on, il signalerait que le locuteur, tout en parlant du bébé et au bébé, s'associe à la situation. Cet effet d'empathie reste sensible dans le texte d'Echenoz: le on de comme on ne possédait plus de représentation de Sylvie, tout en faisant allusion à Fabre et au fils, et à la situation dans laquelle ils se trouvent, associe le narrateur (et le lecteur) au constat.

17 Le renvoi à Fabre et Sylvie à l'aide de simples pronoms dans il s'épuisait à vouloir la décrire toujours plus exactement ne pose aucun problème : il renvoie sans ambiguïté à Fabre, sujet de la phrase précédente et donc beaucoup plus saillant que Paul, et la marque de genre aussi bien que le verbe sélectionnent la mère. La phrase qui suit: Au milieu de la cuisine naquirent des hologrammes est, elle aussi, typiquement thétique. Le verbe, à sémantisme faible, marque simplement l'advenue d'un événement, en l'occurrence d'une série d'événements du type achèvement, et, autre trait caractéristique, le sujet est postposé à la suite d'un circonstant spatial en tête de phrase, mais non détaché. Les constructions de ce type dites « inversions locatives » sont propres à l'écrit et elles mettent l'accent sur le référent du groupe nominal indéfini qui introduit une entité nouvelle. Le nom hologrammes, à consonance savante, contraste de façon assez cocasse avec le lieu au milieu de la cuisine. Le sens technique peut échapper sans que la référence en pâtisse. Quelque connaissance qu'il en ait, le lecteur n'a en effet aucun mal à se représenter de quoi il retourne globalement. Le contexte supplée : on infère que ce doit être des sortes d'images artificielles, d'analogons dont la reconstitution exige de Fabre un travail épuisant pour l'esprit et la vue (en posant sa main sur sa tête, sur ses yeux).

Le Ça ne se rend pas attribué à Fabre est parfaitement ajusté à la situation. Le pronom démonstratif est en plein dans son rôle, très courant, notamment à l'oral, rôle qui consiste à renvoyer à un ou plusieurs référents ou à une situation hautement accessible dans la situation, en les présentant comme saisis directement, indexicalement, avant toute catégorisation. Le référent visé ne fait pour le coup aucun mystère, il s'agit de Sylvie Fabre dont on vient de nous dire que Fabre s'épuisait à vouloir la décrire toujours plus exactement. Mais ça ne fait pas qu'allusion à Sylvie, il renvoie globalement à l'expérience que peut en avoir Fabre, et dont les hologrammes, ne peuvent, comme il le relève, rendre compte correctement. Cet effet apparaît bien quand on compare avec le pronom de troisième personne. Avec elle ne se rend pas, le pronom renverrait à la personne, à l'être humain particulier en tant qu'actant majeur de l'histoire, mais l'indexicalité serait perdue, car le pronom personnel, du fait de la marque de genre, cache toujours un nom qui contrôle sa forme, lequel nom dénote forcément une catégorie. Le pronom démonstratif se prête à un grand nombre d'usages mais il élimine, dans tous les cas, ce détour conceptuel, d'où l'intuition que l'on a, quand il sert à désigner comme ici une personne, que celle-ci est appréhendée comme une sorte de tout inclassable, inséparable 
de l'expérience que l'on peut en avoir. Ça ne se rend pas ne réfère donc pas simplement à la mère, mais à celle-ci telle que vécue par Fabre qui demeure au premier plan.

Fabre continuant à occuper le devant de la scène, le retour à Paul ne peut se faire avec un simple pronom, la redénomination s'impose: souvent ce fut à Paul de déplier le canapé convertible, transformant les choses en chambre à coucher. Les choses surprend : la chose, aurait été compris comme renvoyant anaphoriquement au canapé. Avec les choses, le nom hyperonymique est bien catégorisant, contrairement à ce qui se passe avec le pronom démonstratif, et il élimine les référents animés. Mais le choix d'un nom aussi peu sélectif et descriptif que chose et non, d'un nom dit de catégorie de base, est ressenti comme anormal. Ce choix doit être justifié, notamment par différence avec la pièce (la cuisine ?) qui aurait été non marqué. Le pluriel indique que les choses réfère déictiquement aux entités matérielles accessibles dans la situation, pour les participants, et, pour les lecteurs, au modèle mental qu'ils ont pu construire des lieux à la lecture de ce qui précède (interprétation anaphorique). Dans le contexte, les choses n'est donc pas interprété comme référant universellement à toutes les choses possibles et imaginables, mais comme faisant allusion aux choses figurant dans la zone de l'espace où se trouvent les personnages, autrement dit dans les deux pièces, dont il a justement été précisé qu'elles avaient des fonctions permutables, et que donc elles n'étaient pas vraiment deux pièces bien identifiées, non plus, du reste, comme on l'apprend à ce point du texte, que tout ce qui pouvaient s'y trouver.

Le dimanche et certains jeudis, ils partaient sur le quai de Valmy vers la rue Marseille, la rue Dieu, ils allaient voir Sylvie Fabre. Elle les regardait de haut, tendait vers eux le flacon de parfum Piver, Forvil, elle souriait dans quinze mètres de robe bleue. Le gril d'un soupirail trouait sa hanche. Il n'y avait pas d'autre image d'elle.

20 Le groupe nominal adverbial le dimanche et certains jeudis ferme le cadre ouvert par le soir après le dîner et en ouvre un nouveau qui reste dans le même intervalle temporel. L'interprétation habituelle est maintenue sur tout le paragraphe, avec une distribution différente ; chaque dimanche et seulement certains jeudis. Le distinguo est intriguant : le dimanche est relativement convenu (jour férié), par contre la restriction sur certains jeudis est opaque : convenances personnelles? habitudes familiales? fêtes religieuses? rituels domestiques? En tout cas, des occasions, pour le père et le fils, de se retrouver (ils [...] ils [...] les [...]) et d'emprunter le quai de Valmy. Et, pour le lecteur, d'apprendre pourquoi le fait que l'appartement trouvé par Fabre avait cela de bien d'être situé à proximité de cet endroit.

Cette gratification interprétative n'est que de courte durée puisqu'elle est immédiatement suivie du motif de la visite de Fabre et du fils Paul, qui ne manque pas de surprendre. Comment peuvent-ils en effet aller voir Sylvie qui a disparu dans l'incendie fatal sur lequel débute le texte ? L'inférence qu'ils doivent se rendre sur sa tombe est plus ou moins inévitable dans un premier temps, mais celle-ci est vite défaite en faveur d'une autre interprétation, au demeurant bien accordée avec le sens littéral de aller voir, puisqu'il s'agit pour Fabre et Paul d'aller contempler l'image de Sylvie, son portrait en pied, et en grand, sur le mur d'un immeuble. S'ils la contemplent, elle les regardait de haut, à nouveau au sens littéral (ce qui ne coule pas de source et ne manque pas, comme aller voir de jouer très habilement sur les mots), en grande tenue, dans un geste d'offrande majestueux, mais intéressé (publicité commerciale). 
'histoire pourrait en rester sur le constat impersonnel qu'il n'y avait pas d'autre image d'elle et s'arrêter sur cette scène récurrente qui, comme dans toute bonne narration, vient réparer un manque initial. La suite est celle d'un combat que mènent Fabre et le fils Paul pour lutter contre les dommages que va subir, avec le temps, non pas Sylvie mais son effigie en deux dimensions.

\subsection{La mère et son effigie}

23

Réintroduite dans le récit à la fin du troisième paragraphe, la mère occupe une place centrale dans la suite du texte avec, comme on le verra, quelques éclipses. Sylvie est présente à la fois en tant que personne et en tant qu'effigie, et les mêmes expressions référentielles (explicites ou implicites) peuvent être employées pour référer à l'une ou l'autre de ses deux facettes. Les expressions référentielles et anaphoriques n'offrent en effet pas de formes spécifiques pour signaler que les êtres particuliers dénommés ou dénommables à l'aide d'un nom propre (humains, animés, villes, fleuves, ...) ont changé d'état ou de fonction. Les particuliers que l'on peut dénommer à l'aide d'un nom propre persistent aux changements, leur existence (réelle ou fictive) ne dépendant pas de leurs attributs qualitatifs. Les noms propres sont en effet des désignateurs rigides, arbitraires, qui ne décrivent pas leurs porteurs. L'acte de baptême qui scelle le lien entre le nom propre et son (ou ses) porteur(s) est purement conventionnel, d'où la capacité qu'il a de pouvoir désigner celui-ci (ou ceux-ci) sous toutes leurs apparences. Le prédicat de la phrase dans laquelle figure un nom propre sélectionne une facette d'un particulier, et cette facette peut adhérer à sa personne. C'est le cas, notamment avec les noms propres désignant des êtres humains, lorsque le verbe fait allusion à la dépouille du porteur (Malraux est au Panthéon). Dans les emplois de ce type, où le référent-source (Malraux en tant qu'être vivant) et le référent-cible ne peuvent coexister, le lien est très étroit au point que l'on peut parler de coréférence Malraux $x_{i}$ a été... Il $I_{i} \ldots I_{i . . .}$ Maintenant, $i_{i}$ est au Panthéon. Par contre, lorsque le référent-cible est une représentation en deux ou trois dimensions de la personne (Le docteur Gachet est sur le mur du fond), la prédication sélectionne un référent cible qui peut coexister avec la source et il n'y a plus coréférence, même si la source et la cible restent associés. Il en va a fortiori de même avec les emplois comme George Sand est sur l'étagère de gauche (ses ouvrages) ou Nicky Lauda perd de l'huile (sa voiture) qui ont suscité un grand nombre d'études et de discussions en philosophie du langage et en linguistique.

Pour signaler la différence entre Sylvie Fabre et son effigie, on est obligé d'affecter des indices différents aux expressions référentielles et anaphoriques selon que celles-ci font allusion soit à sa personne soit à sa représentation (cf. Landragin, Tanguy \& Charolles, ce volume) :

§ 3 Le dimanche et certains jeudis, il $s_{j+k}$ partaient sur le quai de Valmy vers la rue Marseille, la rue Dieu, ils $\mathrm{j}_{\mathrm{j}+\mathrm{k}}$ allaient voir Sylvie Fabre $\mathrm{i}$. Elle $_{\mathrm{i}}$, les $_{\mathrm{j}+\mathrm{k}}$ regardait de haut, tendait vers eux $_{\mathrm{j}+\mathrm{k}}$ le flacon de parfum Piver, Forvil, elle $\mathrm{i}_{\mathrm{i}}$, souriait dans quinze mètres de robe bleue. Le gril d'un soupirail trouait $\mathbf{s a}_{\mathbf{i}}$, hanche. Il n'y avait pas d'autre image d'elle $\mathbf{i}^{\text {. }}$

Dans le paragraphe reproduit ci-dessus où Sylvie apparaît pour la première fois sous ses deux visages, le nom propre complet (annoté i') renvoie à son effigie. Mais, comme on l'a relevé dans la partie précédente, les lecteurs qui prennent connaissance du texte pour la première fois, n'ont aucune raison, au moment précis où ils lisent ils allaient voir Sylvie 
Fabre, de penser que Fabre et Paul allaient voir (au sens littéral) le portrait de Sylvie. Vu le contexte précédent, il est possible que les lecteurs infèrent, à ce point du texte, que Fabre et Paul se rendent sur sa tombe, et même qu'ils continuent à interpréter de cette façon le pronom sujet qui suit. La distinction ne s'impose que progressivement, à la lecture des prédicats verbaux qui sont de plus en plus incompatibles avec le fait que ce puisse être sa dépouille qui les regardait de haut. La notation utilisée ne rend donc pas compte de l'interprétation des expressions référentielles en temps réel, elle ne fait que transcrire l'interprétation qui l'emporte. Elle ne donne aucune idée des calculs et ajustements référentiels effectués par les lecteurs qui découvrent le texte. Pour avoir une idée de ces opérations, il faudrait recourir à des moyens expérimentaux, enregistrer les temps de lecture, les mouvements oculaires ou, plus invasif encore, recourir à la neuro-imagerie. Seuls de tels moyens seraient à même d'apporter des indications (indirectes) sur les traitements effectivement mis en œuvre par les lecteurs. Ces méthodes d'investigation sont difficilement compatibles (au moins pour l'instant) avec la lecture d'un texte ordinaire et a fortiori littéraire, mais d'autres moyens moins contraignants, comme le rappel différé sont possibles ${ }^{3}$. Quoi qu'il en soit, l'analyse linguistique ne permet, au mieux, que de repérer les zones du texte où des problèmes dans le calcul de la (co)référence risquent de se poser du fait que les expressions employées sont sousdéterminées.

Sans reprendre l'ensemble des passages où le texte fait allusion à Sylvie, on notera qu'après le cap assez difficile du troisième paragraphe, les transitions entre les deux facettes du personnage se font sans difficultés particulières. Le quatrième paragraphe commence par rappeler dans quelles conditions Sylvie a fini par accepter de servir de modèle pour l'artiste Flers. Dans cette partie aussi bien qu'au début du paragraphe 5 , il n'est question que du modèle et donc de la personne. Les choses se compliquent un petit peu avec la séquence dialoguée qui suit :

Regarde $_{k}$ un peu $\mathbf{t a}_{\mathbf{k}}$ mère $_{i}$,' $\mathrm{s}_{j}$ 'énervait $\mathrm{Fabre}_{\mathrm{j}}$ que ce spectacle mettait en larmes, en rut, selon. Mais $\mathrm{il}_{\mathrm{j}}$ pouvait aussi chercher la scène, $\mathrm{se}_{\mathrm{j}}$ faire franchement hostile à l'endroit de l'effigie ${ }_{\mathrm{i}^{\prime}(\mathbf{e} \text { (i) }}$ contre laquelle $_{\left(\mathrm{ei}^{\mathrm{i}}\right)}$, en écho, rebondissaient ses, reproches $_{\mathrm{j}}$

où le groupe nominal possessif ta mère a deux indices : un sur le déterminant qui renvoie à Paul et l'autre qui réfère à la représentation de Sylvie, sans que pour autant le lien avec la personne soit effacé. À ce stade du texte, le prédicat (regarde) sélectionne clairement l'effigie de Sylvie qui est du reste reprise ensuite par le SN défini l'effigie, puis par le relatif laquelle. L'unicité est marquée par le défini l'effigie, qui cache une forme vide (notée en indice par e) renvoyant à Sylvie en tant que personne. Dans la suite, les renvois à l'effigie impliquent des prédicats qui ne peuvent s'appliquer qu'à sa représentation (Paul visita sa mère sur un rythme plus souple... D'une cabine scellée dans le champ visuel de Sylvie - § 6), et les $\mathrm{SN}$ définis ou possessifs faisant allusion aux parties du corps ou aux vêtements de Sylvie renvoient univoquement à son portrait ([...] la robe bleue, aux pieds de Sylvie - § 6). L'effigie de Sylvie disparait ensuite brièvement pour reparaître au paragraphe 8 où elle occupe le premier plan ([...] son parfum levé par-dessus la charogne, Sylvie Fabre luttait cependant contre son effacement personnel, bravant l'érosion éolienne de toute la force de ses deux dimensions). Elle est encore présente aux paragraphes 9 ([[...] les étages burent Sylvie), puis 10, (Mais à partir des épaules [...] la robe entière [...]. C'était un sépulcre au lieu d'une effigie de Sylvie, on l'approchait d'un autre pas, d'une démarche moins souple). Totalement absente dans les paragraphes 11 et 12, Sylvie réapparait comme effigie au paragraphe 13 ([...] dans un studio situé sous les yeux de Sylvie [...] Selon ses calculs il dormait contre le sourire, suspendu à ses lèvres) 
, et il n'est plus question de sa personne ni de son effigie dans les deux derniers paragraphes, quoiqu'elle soit, comme on le comprend bien, mais sans que cela soit dit, au centre des occupations de Fabre et de Paul.

\subsection{Fabre et le fils Paul}

Dans les deux premiers paragraphes, Fabre, Paul et la mère disparue sont mentionnés, mais Fabre occupe, comme on l'a déjà relevé, une place plus importante que le fils : c'est lui qui a l'initiative, le fils suit, et apprécie. Les deux personnages sont réunis au paragraphe 3 ([...] ils [...] ils [...] (elle) les [...]) et ils réapparaissent, mais chacun de leur côté, et avec la mère, au paragraphe 6 , où c'est Paul, suffisamment séparé de son père, qui est à l'origine des retrouvailles. La suite du texte confirme la prééminence de Paul et le déclin concomitant du père : Paul visite sa mère seul, il suit l'évolution des travaux et surprend son père sur le chantier ( $\$ 9$ ), il constate les dégradations du portrait de Sylvie qui en résultent, cesse de la visiter pendant des semaines, avant de revenir plus tard sur les lieux ( $\$ 10$ et $\S 11$ ) et de monter voir son père dans le nouvel appartement qu'il occupe. À partir du paragraphe 6 , les apparitions des personnages (et des lieux) suivent donc les allers et venues de Paul, les évolutions dans la situation, qui s'étalent sur une période de plus en plus longue, sont rapportées au fur et à mesure de ses visites, Sylvie et Fabre ne sont mentionnés que lorsqu'ils apparaissent.

Paul sert de fil conducteur mais aussi de centre de perception, comme on le voit bien au paragraphe 12, avec la description du nouvel appartement de Fabre: ce sont les impressions de Paul qui sont rapportées, ainsi que les hypothèses qu'il échafaude les revers [qui] avaient dû sévir pendant leur perte de vue. Le constat sur l'état dans lequel se trouve Fabre est introduit par un mais qui trahit aussi le point de vue de Paul : mais Fabre se tenait bien vêtu, ne craignait pas l'eau froide. Ce constat est présenté comme corrigeant une inférence contextuelle (à savoir que l'on aurait pu s'attendre à ce que Fabre soit aussi négligé que les lieux) que l'on ne peut imputer qu'à Paul.

Fabre reprend le dessus au paragraphe 13 dans le discours rapporté (adressé à Paul) retraçant ses démarches pour acheter le studio qu'il occupe désormais et qu'il a choisi parce qu'il était situé sous les yeux de Sylvie qui étaient deux lampes sourdes derrière le mur de droite. L'effigie de Sylvie, qui avait disparue, refait surface dans le discours de Fabre (selon ses calculs il dormait contre le sourire, suspendu à ses lèvres comme dans un hamac), dont le contenu est rapporté indirectement (la voix de Fabre exposait une mission supérieure). Cette voix, déjà présentée comme énervante au paragraphe 11 , se charge de reproches à l'encontre de Paul ( $\$ 5$ ) qui reste insensible à son pathos (une cause auprès de quoi les nerfs $d u$ fils pouvaient faire l'autruche). Pour remettre le fils au premier plan, à la fin de ce paragraphe où Fabre est central, le nom propre s'imposait: Paul partit quand même après vingt minutes. À noter le quand même qui fait implicitement allusion à une norme et signale que celle-ci ne trouve pas à s'appliquer dans les circonstances. Quand même peut être compris de deux façons, selon qu'on le rattache au complément de durée ou à la proposition entière. Dans la première lecture on comprend que Paul aurait dû s'en aller immédiatement ( $v u$ les reproches de Fabre) mais qu'il est resté un certain temps, par respect pour leur auteur. Dans la seconde, on comprend que Paul, convaincu par les propos de Fabre, aurait dû rester, mais qu'il est parti pour une raison supérieure non précisée. La rupture est dans tous les cas brutale et elle tire habilement vers la suite, dont on ne sait pas bien dans quel sens elle peut aller. 
31 Le pronom, au début du paragraphe 14 : Il rassembla ses affaires, ne peut renvoyer qu'à Paul qui vient d'être redénommé et donc remis au premier plan. Le pronom marque, malgré l'alinéa, que la situation dénotée par la première phrase et celle dénotée par la seconde (et revint samedi soir) qui suit immédiatement prolongent celles mentionnées dans le paragraphe précédent. Cette continuité transparaît aussi à travers l'emploi du circonstant temporel samedi soir qui est remarquable. Tout ce que l'on sait au moment où cette expression est employée c'est en effet que Paul a retrouvé son père lors d'une visite quai de Valmy, mais cette visite n'est pas située dans la chronologie : des semaines passèrent avant qu'il revînt quai de Valmy, d'ailleurs accidentellement (\$ 10). Le jour de la semaine où Paul revoit Fabre n'étant pas signalé, il devrait être impossible de faire allusion au samedi faisant suite à cette rencontre à l'aide seulement du nom du jour de la semaine. On s'attendrait à un groupe nominal défini anaphorique le samedi soir (suivant). Les noms de jours de la semaine sans déterminant sont en effet déictiques, leur référence est calculée réflexivement par rapport au moment de leur énonciation, ce qui ne pose aucun problème dans les échanges en situation immédiate où les participants ont accès à ce repère. Comme ce repère fait défaut dans l'emploi qui nous préoccupe, on est obligé de rattacher le nom de localisation temporelle samedi soir à la situation d'échange rapportée dans le paragraphe précédent, et de l'envisager comme une sorte de citation non marquée. Le circonstant force donc, ainsi que le pronom sujet, à comprendre que les actions accomplies par Paul juste après avoir retrouvé Fabre, s'inscrivent dans le prolongement direct de la discussion qu'il a eu avec lui. Ce forçage est discret : il est possible qu'il échappe au lecteur, mais il est sémantiquement motivé. On peut en dire autant du groupe nominal sujet dans Le père avait fait quelques courses, un autre bloc de mousse... qui suit. Le choix de le père plutôt que Fabre fait écho à du fils à la fin de paragraphe précédent et déjà commenté. L'allusion au fait que Fabre avait acheté un autre bloc de mousse donne à penser que Fabre avait anticipé le retour du fils ingrat, malgré la rudesse (au moins textuelle) de son départ.

Si Paul revient au bercail, ce ne peut guère être, présume le lecteur, que pour contribuer à la mission supérieure projetée par Fabre. Et c'est bien ce qui se passe jusqu'à la fin du texte où les deux protagonistes agissent ensemble. Le projet auquel ils se préparent puis s'activent n'est pas explicitement indiqué. Le lecteur doit reconstituer l'intention qui les anime. Le contexte est suffisamment explicite pour que l'on comprenne à quoi ils vont se livrer avant même qu'ils aient commencé. Le but à atteindre ne fait guère de doute, mais il n'est jamais nommé : aucune mention non plus, jusqu'à la fin du texte, de l'effigie de la mère. Seuls Fabre et le fils, coupés du monde extérieur, sont en piste. Ils agissent mais, loin d'occuper le devant de la scène, ils finissent, et c'est un point qui ne peut échapper au lecteur, par se confondre sous la forme d'une personne indistincte, d'un on indéfini qui vient subtilement brouiller la référence.

On apparaît deux fois dans le paragraphe 14, et dix fois dans le paragraphe 15 où il est en plus repris quatre fois par des réfléchis (se) et une fois par un possessif (son gré). Dans le paragraphe 15, on alterne avec ils (ils s'exprimèrent également peu sur la difficulté de leur tâche [...]. Ils mangèrent [...], leurs mâchoires [...]) mais, à la fin du paragraphe, où le texte passe au présent, il n'y plus que on dans une succession de phrases brèves, dont trois de suite avec le verbe gratter :

[...] on a revêtu dès le matin ces larges tenues blanches pailletées de vieille peinture, on gratte et des stratus de plâtre se suspendent au soleil, piquetant les fronts, les cafés oubliés. On gratte, on gratte et puis très vite on respire mal, on sue, $[\ldots]$ 

procès dynamiques, invite à considérer, que on renvoie aussi bien que le pronom personnel au groupe formé par Fabre et Paul. Le fait que la coréférence se maintienne entre ils et on n'est pas douteux mais, comme nous l'avons déjà relevé à propos du on de comme on ne possédait plus de représentations de Sylvie Fabre en commentant le second paragraphe, on peut parfaitement associer d'autres personnes au groupe constitué par Fabre et Paul. C'est ce qui se passe aussi dans le paragraphe 15. Quand on y regarde d'un peu plus près, on s'aperçoit en effet que le on dans d'un tel compte à rebours on peut, avant terme, convoquer à son gré le zéro qui introduit une réflexion plus générale sur les événements en cours, ne fait pas seulement allusion à Fabre et Paul. La transposition avec ils (d'un tel compte à rebours ils purent, avant terme, convoquer à leur gréle zéro) fait ressortir la différence. On généralise, il fait allusion à une personne arbitraire : « qui que ce soit qui se trouverait (comme Fabre et Paul) pris dans un tel compte à rebours, il convoquerait [...] ». Plus exactement, on fait allusion à un actant sujet collectif, humain, indéterminé, lié au prédicat (convoquer) et à la situation (dans un tel compte à rebours), alors que le pronom pluriel les différencie, notamment lorsqu'il s'agit de convoquer le zéro dont l'interprétation ne peut être que distributive (ils purent convoquer chacun à leur gré), et non collective.

Dans les emplois situationnels ou dans le discours rapporté, comme Regarde un peu le soleil qu'on a, dit-il, à la fin du paragraphe 14, on commute avec nous (Fabre et Paul) déictique, mais il inclut aussi potentiellement toute personne qui se trouverait là et qui regarderait le temps qu'il fait. Dans les emplois textuels de on, comme ceux du paragraphe 15 cidessus, où il n'y a plus moyen de faire jouer la procédure déictique, il ne reste que les situations. Ces situations rendent accessibles des agents arbitraires via une formule du type «qui que ce soit qui $\mathrm{V}$ dans l'univers de discours considéré ». L'univers de discours est une notion difficile à définir qui permet de restreindre les mondes possibles. On le voit bien avec les énoncés comme En Angleterre, on roule à droite. On, comme tous les indéfinis est lié par le prédicat de sa phrase d'accueil, toutefois, comme la phrase à une valeur normative et générique, il ne sélectionne pas un individu spécifique, mais une série indéfinie d'individus possibles, tels que chacun « s'il conduit et s'il est en Angleterre, alors il doit rouler à droite ». Lorsque le prédicat est spécifiant comme c'est le cas avec on gratte , qui se prête plus difficilement à une interprétation générique et normative, on tend à se raccrocher, dans un premier temps, au contexte, en l'occurrence à l'action accomplie par Fabre et Paul. L'interprétation qui l'emporte est à nouveau proche de celle qui s'imposerait avec ils. Mais avec on elle ne s'arrêterait pas là, il y aurait, ensuite ou simultanément, élargissement de la référence à d'autres individus accessibles dans le contexte immédiat. Parmi ces individus hautement accessibles il y a, réflexivement, les participants à l'énonciation, en l'occurrence, à l'écrit, l'auteur mais aussi les lecteurs, qui se trouveraient ainsi, bon gré mal gré, impliqués dans l'affaire.

Dans tous les cas, il y aurait mise en avant de la situation: quand on lit une phrase commençant par on, on n'a pas déjà un référent en tête que on servirait simplement à désigner. Le calcul de la référence de on n'est possible qu'à la fin de la phrase qui l'héberge, ce qui veut dire que, au terme de l'interprétation, c'est la situation qui prédomine. On en a l'exemple dans le dernier paragraphe de L'Occupation des sols où l'accent se déplace sur les actions elles-mêmes, et où les personnages passent au second plan : le père et le fils, qui agissent évidemment, mais aussi, en sourdine, Echenoz et nous. Fabre et Paul, tel un commando en mission grattent et regrattent dans le plus grand secret, ce qu'ils grattent importe peu, non plus du reste que ce soit eux qui grattent, ni 
pourquoi ils le font. Tout ce qui ressort finalement, c'est qu'il y a grattage, un peu comme ce qui se passe avec le trou dont il est question au paragraphe 9 : il y a le trou. Il y eut le trou . On suffit aussi pour enclencher toute une chaîne (en l'occurrence, d'événements), il s'en trouve toujours un qui, comme l'explique Echenoz, scelle ce qui le précède, colore ce qui va suivre.

Ce glissement des personnages aux actions, que l'on observe à la fin de L'Occupation des sols, est en fait soigneusement préparé et encadré. Il est amorcé dès le paragraphe 14 où Fabre et Paul commencent à perdre de leur saillance au profit des événements. De retour dans le logis paternel, Paul non plus que le père pourtant si disert, n'occupent en effet le devant de la scène: Nul ne raconta rien de ces dernières années, rien ne s'évoqua sous l'ampoule nue. Le calcul de la référence de nul et rien, qui sont des pronoms indéfinis (respectivement +/-humain et -humain) passe par les mêmes ressorts que ceux présidant à l'établissement de la référence de on. À une différence majeure près qui est que nul et rien signalent que le croisement du prédicat et du contexte ne sélectionne aucune entité à même d'accomplir l'action mentionnée (nul) non plus que l'objet de celle-ci (rien) : il n'y a aucune personne pour raconter, ni Fabre, ni Paul, ni x, et par voie de conséquence aucune chose qui se trouve évoquée. Les énoncés, complètement thétiques de ce type, ne parlent que de la situation, ils disent simplement qu'il n'y a pas eu un seul événement du type indiqué. Ils ne font allusion ni aux personnages, ni à qui que ce soit que l'on pourrait leur associer. On n'est pas loin, avec les phrases négatives de ce genre, des énoncés météorologiques, et ce n'est probablement pas un hasard si L'Occupation des sols s'achève définitivement sur : il commence à faire terriblement chaud.

\subsection{L'artiste Flers, l'usager, Jacqueline et les autres}

\subsubsection{L'artiste Flers}

L'artiste Flers occupe une place centrale dans le paragraphe 4. Il est repris une fois au début du paragraphe suivant, puis disparaît de l'histoire. Son rôle est limité mais assez crucial pour la compréhension, dans la mesure où il entre en scène juste après que l'on a appris que Fabre et Paul allaient voir Sylvie quai de Valmy. Le paragraphe suivant explique la raison pour laquelle elle se trouve en cet endroit sur un panneau publicitaire couvrant la façade d'un immeuble. Étant donné l'étrangeté des faits, l'explication, n'est probablement pas inutile pour les lecteurs qui, à la suite du paragraphe précédent, pourraient se demander s'ils ne se sont pas trompés quelque part dans le texte.

L'auteur de l'effigie de Sylvie est introduit par un groupe nominal défini précisant sa profession suivi de son nom propre apposé : l'artiste Flers. Ce type d'introduction n'est pas courant mais il se comprend bien dans le contexte. L'indication de la profession est très coopérative car on saisit d'emblée le lien de cohérence avec ce qui précède. L'emploi du seul nom propre était assez difficile. Flers n'étant pas un nom connu, le nom patronymique seul aurait connoté un usage autonymique sociolectal et donc suggéré un lien avec Fabre et Paul (comme avec Fabre commenté dans la première partie). Le nom propre complet aurait été requis et le verbe qui suit (l'avait représentée) n'aurait laissé aucun doute sur son rôle.

L'artiste Flers est repris dans le même paragraphe avec la même expression, ce qui est plus curieux : l'artiste aurait suffi, ou bien Flers seul, comme c'est du reste le cas au début du paragraphe 4. Ce type de dénomination est assez rare, il semble réservé à certains usages, 
notamment au théâtre, où les personnages sont désignés par leur nom de rôle (la servante) suivi de leur nom (la servante Zerline) notamment s'il y en a plusieurs assumant les mêmes fonctions. Il n'est pas exclu de voir dans cet usage, une anticipation du fait qu'à la fin de l'histoire les personnages principaux tendent à s'effacer, au profit des actions qu'ils accomplissent et donc de leur rôle.

\subsubsection{L'usager}

41 L'usager qui apparaît dans le paragraphe 7 donne naissance à une chaîne de référence très nourrie puisqu'il est ensuite repris treize fois en quelques lignes. Introduit comme l'usager, il est repris deux fois, juste après, à l'aide du même groupe nominal, puis par un pronom sujet qui consacre définitivement son statut de personnage. Ce personnage est assez curieux, à plusieurs titres.

42 Usager est un nom relationnel (contrairement à artiste) : il est dérivé du nom usage, luimême déverbal de user qui appelle un complément. On use de quelque chose et si l'on est un usager, on ne peut l'être que d'une certaine chose, quelle qu'elle soit. Le nom usager étant relationnel (ou syncatégorématique), le calcul de la référence du groupe nominal défini singulier l'usager implique qu'on le raccroche à une entité présumée accessible dans le contexte, et susceptible de servir à certains usages. La sélection de cette entité n'est pas bien difficile dans le texte: il s'agit de l'usager de l'espace vert, qui formait un gazon subsidiaire aux pieds de Sylvie introduit au paragraphe 6, et repris comme l'espace au début du paragraphe 7 (on laissait l'espace dépérir).

Une fois ce lien établi, il reste encore à calculer la référence de l'usager. L'entité dont il est question est présentée comme unique de son type. Les espaces verts étant stéréotypiquement associés à des personnes qui en profitent, le pluriel les usagers serait compris et traité sans difficulté comme une anaphore associative de l'espace vert. Avec l'usager au singulier, les choses sont moins simples. Le singulier n'est possible qu'avec une valeur générique (l'usager en général) où il désigne non pas un ensemble de personnes associées mais un type qui est une entité plus abstraite que l'on saisit comme s'opposant à d'autres types. Pour se rendre compte de cette différence sémantique qui est assez subtile il n'est qu'à penser aux magasins, et aux clients (au pluriel) qui, pour le sens commun (et la langue) vont avec. Un énoncé comme Dans ce magasin, les clients sont rois ne dit pas tout à fait la même chose que Dans ce magasin, le client est roi. Dans tous les cas, l'interprétation passe par le magasin, mais avec le pluriel les clients sont mentionnés en tant simplement qu'ils fréquentent les lieux, comme prévu par le stéréotype. Avec le singulier, ils sont envisagés un peu différemment comme formant une catégorie de personnes associées au lieu mais qui se différencie (et que l'on veut différencier) d'autres catégories de personnes le fréquentant aussi, comme le personnel. Le client fait allusion à un rôle spécifique, le client est roi parce qu'il est client, parce qu'on le traite en client, pas parce qu'il se trouve là. L'usager d'Echenoz s'explique de la même façon. Le groupe nominal introduit une sorte de consommateur d'espace vert type, dont les prérogatives sont prévues par le règlement des lieux et qui peut faire valoir certains droits, comme c'est du reste le cas dans L'Occupation des sols, où l'usager, volontiers, s'offense de ces pratiques.

Une fois introduit dans le texte sous les traits singuliers que l'on vient d'indiquer, l'usager peut ensuite parfaitement être repris, comme c'est effectivement le cas :

Heurté, l'usager boycotte cet espace rayé du monde chlorophyllien, n'y délègue plus sa descendance, n'y mène plus déféquer l'animal familier. Le trouvant un matin 
barré d'une palissade, il cautionne cette quarantaine l'œil sec, sans se questionner

sur son initiative ; son cœur est froid, sa conscience pour soi. qui criait Fabre Fabre, elle réapparaît ensuite avec son prénom. Jacqueline figure dans le discours direct, non marqué par les guillemets, mais parfaitement clair. Le prénom n'est pas anaphorique, la coréférence est établie par le biais du discours rapporté. Comme le prénom figure dans la réponse que Fabre adresse à la femme qui vient de l'appeler, le lecteur infère qu'il s'agit de la même personne et ce faisant il apprend qu'elle se prénomme Jacqueline, ce qui présuppose que Fabre la connaît bien, comme le confirme aussi le fait qu'il la tutoie. Tout cela est parfaitement normal comme du reste sa reprise ensuite à l'aide du défini la femme s'éloignait. Son départ est décrit du point de vue de Paul (cf. le verbe andatif s'éloigner), le commentaire on ne sait pas qui c'était est imputable à celui-ci, mais l'emploi de on oblige à nouveau à élargir la référence, notamment au lecteur qui ne peut manquer lui aussi de constater qu'il ne sait pas qui elle est, et ce qu'elle vient faire là.

question se pose d'autant que le contenu de l'échange avec Fabre est très énigmatique. Ce point n'a rien à voir avec la référence, qui, comme on vient le souligner ne pose pas problème, mais il mérite d'être souligné. J'ai du lait, annoncé triomphalement par Jacqueline, n'a aucune raison d'être interprété, dans un premier temps, autrement que comme signifiant «j'ai acheté du lait», et par inférence contextuelle «je peux t'en apporter un peu ». La réponse de Fabre Tu simules Jacqueline, quelle qu'on la comprenne, se raccroche difficilement à cette interprétation. Il est possible que les lecteurs en restent à ce simple constat d'incongruité. Mais, comme il est toujours difficile d'admettre que quelqu'un puisse répondre n'importe quoi à une personne qui vient de lui adresser la parole, on peut s'attendre à ce que les lecteurs essaient de restituer, par inférence contextuelle, un lien de cohérence entre les propos échangés. Cette reconstitution n'est pas aisée. Une des possibilités pourraient être de retraiter J'ai du lait comme signifiant 
«j'ai une montée de lait, je suis enceinte», à la suite que quoi la réponse de Fabre se comprendrait mieux «c'est faux tu simules ». Est-ce que les lecteurs font de tels calculs et se livrent à ce genre d'ajustement? Impossible de répondre à cette question.

\subsubsection{Les autres} (notamment le verbe) et le contexte plus large font que cette interprétation étroite l'emporte d'emblée, et bloque toute velléité d'élargissement à d'autres personnes. Partant de là, il est tentant de considérer que le second on qui apparaît juste après dans la même phrase graphique réfère lui aussi à Fabre et Paul, d'autant que, comme le texte vient de l'indiquer, ils avaient l'habitude de visiter le chantier. L'interprétation étroite a donc toutes les chances de se maintenir, par économie. Mais ce n'est pas certain : le second on se prête à une interprétation plus large, incluant en plus toute personne susceptible de passer le long du chantier. Le problème se pose à nouveau, avec le on qui suit. Le prédicat se mettre à démolir exclut Fabre et Paul, aussi bien que toutes les personnes que l'on pourrait inclure dans l'ensemble dénoté par le second on, de sorte qu'il n'y a pas coréférence entre les trois pronoms. La référence de on doit être calculée à chacune de ses occurrences en fonction du contexte large et étroit. On n'est jamais anaphorique, mais il n'exclut pas qu'il puisse y avoir coréférence, ce qui arrive notamment dans les emplois en situation où il commute avec tu/vous (Alors, on est fatigué, on ferait bien une petite sieste) ou nous (alors on y va, on va finir par être en retard). Est-ce que les lecteurs s'attendent à ce que plusieurs on successifs coréfèrent? Nul ne le sait. Le fait que Grevisse (Le bon usage, § 725) relève que « les grammairiens n'admettent pas qu'une même phrase contienne plusieurs on désignant des personnes différentes ou des groupes différents » et qu'il juge peu clairs des emplois comme on vint dire à $M^{m e}$ Kerkaradec qu'elle était servie, et l'on passa dans la salle à manger suggèrent qu'il pourrait bien en aller de la sorte, mais on ne dispose pas de données comportementales sur le sujet.

\section{La référence aux lieux}

Dans cette partie, nous nous intéresserons à l'expression de l'espace dans L'Occupation des sols en tentant de répondre à deux questions. Comment est exprimée la référence à l'espace dans ce texte, et en particulier la référence aux lieux (immeuble Wagner, espace 
vert, etc.) ? L'importance des lieux dans la narration - importance que suggère le titre - at-elle un corollaire linguistique identifiable?

\subsection{Expression linguistique de l'espace dans L'Occupation des sols}

52 Nous évoquerons d'abord les différents moyens disponibles pour exprimer la référence à l'espace en français, en renvoyant à la typologie établie par Andrée Borillo (1998) et en illustrant brièvement celle-ci par des exemples tirés du texte. Nous nous focaliserons ensuite sur la référence aux lieux, dont il est question dans l'article de Storme et Storme ci-après.

\subsubsection{Généralités}

53 Un récit semble difficilement concevable sans un espace dans lequel se situent les événements qu'il narre. On peut même imaginer que toute phrase dénotant un événement va entraîner une présupposition d'existence d'un lieu dans lequel cet événement se déroule. Par exemple, la phrase Jean a couru le matin du 31 décembre 2012 présuppose l'existence d'un lieu dans lequel l'action de courir a été réalisée le matin du 31 décembre 2012 par l'individu unique accessible dans le contexte d'énonciation qui porte le nom de Jean. Ce lieu peut être dénoté par une expression que l'on dira spatiale : Jean a couru dans [le jardin de Paul] le matin du 31 décembre 2012. Le jardin de Paul constitue ici un repère par rapport auquel l'événement dénoté par le reste de la phrase est situé ; la nature de ce rapport est quant à elle dénotée par la préposition dans.

Il ressort de cette brève introduction que la référence à l'espace est typiquement exprimée par des syntagmes nominaux compléments de prépositions. Ces syntagmes nominaux dénotent des référents qui ont une extension dans une, deux ou trois dimensions (la ligne, la surface, le volume,[...]) ; les prépositions codent quant à elles une relation dans l'espace (dans, sur, sous, hors de [...]) et peuvent être conçues comme des expressions dénotant des fonctions qui associent des référents à des repères. Dans la phrase Jean est à Paris, la préposition à met en relation le référent «Jean » et le repère « Paris » en précisant que le premier se trouve à l'intérieur du second; le morphème de présent contenu dans est indique que cette relation d'inclusion est vraie au moment de l'énonciation.

Cependant, comme l'a montré Borillo (1998) entre autres, la référence à l'espace n'est pas l'apanage de certaines catégories grammaticales, comme les noms et les prépositions. En réalité, des lexèmes de toutes les catégories grammaticales concourent à coder la référence spatiale : noms et syntagmes nominaux dénotant des référents spatiaux (le quai de Valmy, le flanc d'un immeuble [...]), prépositions et syntagmes prépositionnels (sous une cheminée de briques, sur le quai de Valmy, dans un cartouche, au cour d'une cour dallée, après l'entrée), adjectifs et syntagmes adjectivaux (proches du quai de Valmy, bardé de balconnets incurvés), verbes et syntagmes verbaux exprimant des localisations statiques (surplomb[er] un petit espace vert rudimentaire, former le coin de la rue [...]) ou dynamiques (l'immeuble allait atteindre le ventre de sa mère), adverbes et expressions adverbiales (en haut). 


\subsubsection{Référents spatiaux, chaîne de référence et repère : l'exemple de l'immeuble Wagner}

Dans l'article ci-après sur la représentation de l'espace chez le lecteur (Storme et Storme) les auteurs se sont intéressés aux référents spatiaux, tels l'immeuble Wagner ou le quai de Valmy. Aussi semble-t-il opportun de s'arrêter sur l'identification de ces référents spatiaux et sur la façon dont ils sont exprimés dans le texte.

D'après Borillo (1998, p. III), certains noms ont un trait sémantique [+spatial] « caractérisant la vision que nous avons des entités physiques auxquelles ils réfèrent et de leur manière d'occuper l'espace à trois dimensions dans lequel nous avons appris à nous repérer." Cette définition pourrait permettre d'élaborer une liste exhaustive des référents spatiaux du texte si la notion de trait [+spatial] n'était pas aussi commune : en effet, tout objet concret occupant une certaine portion d'espace, toute mention de cet objet pourrait constituer une expression spatiale, y compris des noms propres comme Paul. Nous avons néanmoins établi une telle liste, qui comporte bien sûr une part d'arbitraire, et avons dénombré 48 référents spatiaux dans le texte, incluant les rues, le canal, les bâtiments et certains objets afférents à ces bâtiments (le porche de l'immeuble Wagner, la palissade qui entoure l'espace vert...) ou contenus dans ces bâtiments (l'appartement qui a brûlé, le studio...).

Chacun de ces éléments spatiaux va être rappelé un plus ou moins grand nombre de fois au cours de la narration. L'immeuble Wagner, qui est un des éléments les plus cités dans le texte et les plus rappelés par les lecteurs (cf. Storme et Storme ci-après), donne naissance à des chaînes de référence, de façon attendue.

La première mention de ce référent est faite au moyen d'un syntagme nominal indéfini, un immeuble (\$ 4): L'artiste Flers l'avait représentée sur le flanc d'[un immeuble] $]_{i}$ juste avant le coin de la rue. L'article indéfini est typique lorsqu'il s'agit d'introduire un référent encore inconnu. Ce référent "flambant neuf» est situé dans le repère qui a été défini au paragraphe précédent, à savoir le quartier du quai de Valmy, par le syntagme prépositionnel juste avant le coin de la rue. Le syntagme nominal défini la rue, dans lequel l'article a valeur anaphorique, renvoie à une rue citée précédemment dans le paragraphe 3 , sans que soit encore précisé de quelle rue il s'agit - le quai de Valmy, la rue Marseille ou la rue Dieu? De façon attendue, le référent qui vient d'être introduit et qui est donc connu est dénoté par un syntagme nominal défini, l'immeuble, dans la phrase qui suit: [L'immeuble] $]_{i}$ était plus maigre et plus solide, mieux tenu que les vieilles constructions qui se collaient en grinçant contre [lui $]_{i}$, terrifiées par le plan d'occupation des sols. Le pronom anaphorique lui pourrait grammaticalement renvoyer à autre chose que l'immeuble ; mais puisque dans le cotexte il s'agit du référent le plus saillant (l'expression référentielle l'immeuble est en effet sujet de la phrase principale) et le plus pertinent (une lecture où lui renverrait à l'autre référent dénoté par un nom masculin singulier saillant dans le contexte, à savoir l'artiste Flers, donnerait lieu à un non-sens), le lecteur doit aussitôt coindicer lui et l'immeuble. Dans la suite du paragraphe, l'anaphore pronominale dans le syntagme nominal [son $]_{i}$ porche maintient la chaîne de référence à cet immeuble.

L'artiste Flers l'avait représentée sur le flanc d'[un immeuble $]_{i}$, juste avant le coin de la rue. [L'immeuble] $]_{i}$ était plus maigre et plus solide, mieux tenu que les vieilles constructions qui se collaient en grinçant contre [lui $]_{i}$, terrifiées par le plan d'occupation des sols. En manque de marquise, [son] $]_{i}$ porche saturé de moulures portait le nom (Wagner) de l'architecte-sculpteur gravé dans un cartouche en haut 
à droite. Et le mur sur lequel, avec toute son équipe, l'artiste Flers avait peiné pour figurer Sylvie Fabre en pied, surplombait un petit espace vert rudimentaire, sorte de square sans accessoires qui ne consistait qu'à former le coin de la rue. s'agit de l'immeuble qui est construit à l'emplacement de l'espace vert à partir du paragraphe 9. En effet, les parties de ce référent (les fondations, la superstructure, les étages) sont nommées avant que ne soit nommé le référent lui-même (l'immeuble), à l'inverse de ce qui s'était passé avec l'immeuble Wagner. Certes, cela pourrait être dû au fait que, contrairement aux autres référents, ce référent-là ne préexiste pas aux événements de la narration : il est construit pendant le temps de la narration. Mais on aurait pu s'attendre de façon tout à fait naturelle à trouver, en tête du paragraphe 9, une phrase programmatique du type : On construisit un immeuble à la place de l'espace vert. Au lieu de cela, le processus de construction est évoqué de façon très progressive et très énigmatique. Le narrateur introduit la palissade au paragraphe 7, sans préciser qu'il s'agit d'une palissade sensée clore un chantier; puis, au paragraphe suivant, il évoque l'affichage d'un permis de construire et le creusement des fondations de l'immeuble de façon très absconse ; la construction des fondations et des étages semble quant à elle se produire sans qu'aucun agent humain n'intervienne :

Tendu sur des piquets rouillés, du ruban rouge et blanc balisait le théâtre. Les fondations enracinées, toutes les matières premières livrées, on lança la superstructure et de nouvelles planches neuves traînèrent un peu partout, gainées d'un grumeau de ciment. Les étages burent Sylvie comme une marée. 
Tous les sujets de ce passage renvoient à des objets, à l'exception du on qui réfère à une instance humaine très indéfinie. Tout se passe comme si les éléments du décor devenaient eux-mêmes des actants.

\subsection{Rôle narratif de l'espace}

Comme nous l'avons entrevu dans la partie précédente, les référents spatiaux ont un rôle tout à fait central dans cette histoire. Cette particularité narrative des lieux a un corollaire linguistique assez sensible: les référents spatiaux sont associés de façon « déviante » à des prédicats qui entraînent des présuppositions d'animation (animacy).

\subsubsection{Référents spatiaux et narration : les lieux comme actants}

Le titre de la nouvelle L'Occupation des sols et l'expression le plan d'occupation des sols (cf. § 4) définissent assez bien ce qui est le ressort de l'intrigue: la façon dont la reconfiguration d'un quartier va perturber la vie d'un père et son fils et, par là, les contraindre à agir en conséquence. L'effigie de Sylvie est ici centrale, puisque c'est elle qui, par sa double appartenance au plan des lieux et des personnages, va permettre le relais entre une simple histoire de construction de bâtiments et un drame familial.

On peut d'ailleurs mettre en parallèle la trame narrative ${ }^{4}$ et l'évolution des lieux. On remarque que les étapes de la narration coïncident avec les étapes de l'évolution des lieux.

Tableau 1 - Étapes de la narration et étapes de l'évolution des lieux.

\begin{tabular}{|l|l|l|}
\hline$\S 1-5$ & $\begin{array}{l}\text { Situation initiale : Fabre et son fils vont voir de temps en } \\
\text { temps l'effigie de Sylvie peinte sur l'immeuble Wagner }\end{array}$ & $\begin{array}{l}\text { Étapes de l'évolution des } \\
\text { lieux }\end{array}$ \\
\hline$\S 6-10$ & $\begin{array}{l}\text { Nonstruction d'un nouveau bâtiment à côté de l'immeuble } \\
\text { Wagner }\end{array}$ & $\begin{array}{l}\text { lestruction du vieil } \\
\text { nouveau bâtiment } \\
\text { Dépérissement de l'espace } \\
\text { vert } \\
\text { Construction d'un nouvel } \\
\text { immeuble }\end{array}$ \\
\hline § & $\begin{array}{l}\text { Résolution (en suspens) : Fabre et son fils entreprennent de } \\
\text { gratter le mur qui couvre l'effigie pour la rendre à nouveau } \\
\text { apparente }\end{array}$ & $\begin{array}{l}\text { Début de l'entreprise de } \\
\text { grattage dans le studio }\end{array}$ \\
\hline $10-15$
\end{tabular}

L'importance des lieux est aussi numérique: nous avons relevé 48 référents spatiaux mentionnés par 93 expressions spatiales. Certains paragraphes sont saturés de descriptions de l'espace, qui semblent n'avoir aucune justification narrative évidente a priori : ainsi la description du canal au paragraphe 12, transformé en un dépotoir dont le 
narrateur semble prendre plaisir à énumérer les composants, la description minutieuse de l'espace vert à l'abandon aux paragraphes 7 et 8 et enfin l'évocation énigmatique du trou où sont construites les fondations du nouvel immeuble au paragraphe 9 .

\subsubsection{Référents spatiaux, syntaxe et sémantique : les lieux comme sujets/agents} également perceptible au niveau syntaxique et sémantique: sur les 93 occurrences d'expressions dénotant un lieu ou un référent attenant à un lieu relevées, 23 occupent en effet la position de sujet d'une proposition à un temps fini ${ }^{5}$ (ex. au $\S 8$ : [La palissade] se dégraderait à terme) ou d'une participiale ${ }^{6}$ (ex. au $\$ 6$ : [l'autre flanc du Wagner] se trouvant heureusement protégé par la pérennité de l'espace vert), ce qui semble assez remarquable étant donné le rôle a priori plutôt secondaire des notations de lieux dans un récit. Certes, la plupart de ces occurrences appartiennent à des passages descriptifs à l'imparfait (14 imparfaits, 3 passés simples, 2 plus que parfaits, 2 conditionnels, 2 participes) ; cependant, les prédicats associés à ces expressions ne sont pas toujours les prédicats auxquels on pourrait s'attendre dans la description d'un espace. Ainsi, les deux bâtiments qui encadrent l'immeuble Wagner, sont dits solidaires, gardes du corps du Wagner préservé (\$ 10); le terre-plein dans la cour du nouvel immeuble est dit prédi[re] le retour de la végétation trahie (\$11). Les présuppositions d'animation concernent particulièrement les prédicats qui sont prédiqués de l'effigie de Sylvie qui, comme cela a été mentionné plus haut, est un référent qui est tantôt traité comme humain, tantôt comme inanimé. Dans les exemples suivants, regarder, lutter et braver sont des verbes qui sélectionnent comme sujets des syntagmes nominaux dénotant des agents ; or il est clair ici que ces expressions renvoient à la représentation de Sylvie et non à Sylvie elle-même.

[Elle] les regardait de haut, tendait vers eux le flacon de parfum Piver, Forvil, elle souriait dans quinze mètres de robe bleue. (\$ 3 )

Son parfum levé par-dessus la charogne, [Sylvie Fabre] luttait cependant contre son effacement personnel, bravant l'érosion éolienne de toute la force de ses deux dimensions. (\$ 8)

Cette humanisation des référents spatiaux est particulièrement prononcée dans le paragraphe 9: [Les étages] burent [Sylvie] comme une marée. Paul aperçut Fabre une fois sur le chantier, [l'immeuble] allait atteindre [le ventre de sa mère]. L'emploi du verbe boire avec un sujet inanimé est métaphorique dans la mesure où boire sélectionne un sujet agent; l'emploi de couvrir ([Les étages] couvrirent [Sylvie]) aurait été moins marqué dans la mesure où couvrir peut sélectionner un sujet cause ([Les nuages $]_{\text {cause }}$ couvrirent l'horizon) autant qu'un sujet agent ([Maman $]_{\text {agent }}$ couvrit mes livres). L'utilisation du nom propre Sylvie et du syntagme nominal le ventre de sa mère pour renvoyer à une image et à une partie de celleci est métonymique ; une formulation plus neutre aurait pu être la suivante: [Les étages] burent [l'image de Sylvie]. Cette conjonction de déviances sémantiques n'est pas surprenante à cet endroit du texte: il s'agit en effet du nœud de l'intrigue, dont le caractère dramatique se trouve ainsi souligné.

\section{Conclusion}

Dans les analyses qui précèdent nous nous sommes focalisés sur la façon dont les lecteurs de L'Occupation des sols pouvaient traiter les expressions référentielles renvoyant à des

Revue Sciences/Lettres, 3 | 2015 
humains et à des zones de l'espace. Ces analyses vont de pair avec celles présentées, dans ce volume, par Fuchs, Le Goffic et Maillard sur les situations et leurs relations aspectuotemporelles. Les situations sont des entités qu'il faut d'abord repérer puis suivre, dans la chronologie, au même titre que les personnages, les lieux et les bâtiments qui les occupent. Dans un cas comme dans l'autre, on a affaire à des sortes de choses. Les personnages et les lieux participent aux situations, ils persistent plus ou moins aux changements temporels, ce qui ne manque pas, comme on l'a relevé, de poser des problèmes, en particulier avec Sylvie et les bâtiments.

Nous avons commencé par examiner comment les personnages et les lieux étaient introduits et repris dans l'incipit, puis nous avons élargi l'analyse jusqu'à la fin de texte, en essayant de suivre leur destin référentiel, ainsi que celui des autres personnages et lieux apparaissant ensuite.

Pour ce qui est des personnages, la mère, Fabre et le fils Paul qui sont installés dès les premières lignes, ne sont guère concurrencés dans la suite, si ce n'est, de façon circonscrite, par l'artiste Flers, l'usager et Jacqueline. L'artiste Flers est crucial pour la cohérence, il arrive à point pour comprendre comment Fabre et Paul peuvent encore visiter Sylvie après qu'elle a disparu dans l'incendie liminaire. L'apparition de l'usager, puis celle de Jacqueline est liée aux aléas des allers et venues de Paul. Comme ces personnages ne jouent aucun rôle dans la suite des événements, les développements dont ils font l'objet semblent disproportionnés (pour l'usager) et très énigmatiques (pour Jacqueline), comme si l'auteur s'emballait sur autre chose. Il en va de même, et de façon peut-être encore plus nette, du côté de l'espace, avec l'évocation minutieuse, pour ne pas dire maniaque, des objets traînant dans le canal asséché. Sans rompre la narration, ces excursions la déstabilisent subtilement. Elles donnent aussi à penser que celle-ci pourrait bien n'être qu'un prétexte pour parler d'autre chose, sans que l'on arrive à comprendre précisément de quoi il retourne.

Si le contact avec les personnages centraux n'est jamais perdu, ce qui les menace c'est leur progressif effacement en tant qu'actant. Cet effacement est scellé d'avance pour Sylvie qui, ayant disparu dans l'incendie, n'agit plus qu'à travers son image. Mais il gagne aussi progressivement Fabre et le fils Paul. Leur effacement n'est lisible, comme beaucoup d'autres singularités de la référence notées au passage, qu'à travers des formes d'expression qui sont subtilement orientées et qui sont d'autant plus redoutables que l'on a du mal à préciser leurs effets de sens. Il en va ainsi, comme on l'a relevé, de l'abondance des on dans le dernier paragraphe. Cette abondance ne peut pas ne pas frapper et elle est trompeuse car les on renvoyant simplement au père et au fils, qui sont clairs référentiellement, apparaissent parmi d'autres qui le sont beaucoup moins parce qu'ils figurent dans des énoncés qui ne sélectionnent pas un ou plusieurs personnages déjà mentionnés. Ces on problématiques tendent à prendre une valeur inclusive qui élargit la référence, jusqu'à inclure le lecteur. Lorsque le verbe est sans complément comme avec les on gratte du dernier paragraphe, l'énoncé prend très vite une valeur thétique : l'accent est porté sur l'action elle-même, sur son advenue, sur le fait qu'elle a lieu, comme si elle survenait indépendamment de la volonté et des buts des actants qui l'accomplissent. Ces effets de sens n'ont, linguistiquement, rien d'étonnant. Ils découlent assez directement des instructions référentielles encodées par le pronom indéfini humain on qui occupe une place singulière dans le système des pronoms du français. Ces effets de sens, difficiles à paraphraser, ne font probablement qu'affleurer dans la «conscience» des lecteurs, au moins en première lecture, mais cela n'affecte en rien leur force. 
Cette problématique de l'effacement se retrouve avec les sols et les constructions. L'immeuble Wagner sur lequel figure l'effigie de Sylvie occupe une place centrale dans les lieux. Il sert de repère pour la construction d'une représentation des autres bâtiments qui le jouxtent peu à peu et il subsiste aux bâtiments qu'on lui adjoint successivement mais avec lesquels il finit par se confondre. Cette évolution n'est pas trop difficile à suivre, au moins globalement, sauf qu'elle se traduit par la disparition progressive de l'effigie de la mère (les étages burent Sylvie comme une marée), et que le lecteur a besoin, pour le coup, de se représenter assez précisément l'emplacement de la façade sur laquelle elle se trouve. Cela, qui oblige déjà à certains calculs sur la disposition respective des constructions, ne va pas forcément de soi mais, surtout, cela conduit à un résultat qui est ontologiquement paradoxal, puisqu'il s'agit in fine de se représenter un emplacement qui n'est plus perceptible. Cette zone, devenue théorique, où l'effigie de Sylvie doit continuer à exister, n'est plus accessible qu'à des experts: Fabre et le fils Paul, évidemment, mais aussi les lecteurs... qui doivent mouiller la chemise avec eux pour comprendre de quoi il retourne en fin de compte à la fin de l'histoire. De là à supposer que le texte a lui aussi un sens caché qu'il faudrait gratter, il n'y a qu'un pas que bien entendu, par espièglerie, Jean Echnoz n'invite pas à franchir: on gratte, on gratte et puis très vite on respire mal, il commence à faire terriblement chaud.

\section{BIBLIOGRAPHIE}

Apothéloz, Denis, Rôle et fonctionnement de l'anaphore dans la dynamique textuelle, Genève, Librairie Droz, 1995.

Borillo, Andrée, L'Expression de l'espace en français, Paris, Ophrys, 1998.

Bosveld-De Smet, Léonie, Van Petéghem, Marleen et Van De Velde, Danièle, De l'indétermination à la qualification : les indéfinis, Arras, Artois Presses Université, 2000.

Charolles, Michel, La Référence et les expressions référentielles en français, Paris, Ophrys, 2002.

Corblin, Francis, Indéfini, défini et démonstratif, Genève, Droz, 1987.

-, Les Formes de reprise dans le discours. Anaphores et chaînes de référence, Rennes, Presses universitaires de Rennes, 1995.

Cornish, Francis, Anaphora, Discourse, and Understanding. Evidence from English and French, Oxford, Oxford University Press, 1999.

-, Anaphoric Relations in English and French. A discourse perspective, London, Croom Helm, 1986.

Flaux, Nelly, Van De Velde, Danièle et De Mulder, Walter, Entre général et particulier : les déterminants, Arras, Artois Presses Université, 1997.

Gary-Prieur, Marie-Noëlle, Grammaire du nom propre, Paris, PUF, 1994.

Kleiber, Georges, L'Anaphore associative, Paris, PUF, 2001.

Kesik, Marek, La Cataphore, Paris, PUF, 1989. 
Kleiber, Georges et Tyvaert, Jean-Emmanuel (éd.), L'Anaphore et ses domaines, Paris, Klincksieck, 1990.

Kleiber, Georges, Problèmes de référence : descriptions définies et noms propres, Paris, Klincksieck, 1981.

-, Nominales, Paris, Armand Colin, 1994.

-, Anaphores et Pronoms, Bruxelles, Duculot, 1994.

Leeman, Danielle, Les Déterminants du nom en français - syntaxe et sémantique, Paris, PUF, 2004.

Martin, Robert, Pour une logique du sens, Paris, PUF, 1983.

Schnedecker, Catherine, Nom propre et chaînes de référence, Paris, Klincksieck, 1997.

\section{NOTES}

1. Voir Fuchs, Le Goffic et Maillard, ce volume.

2. Les analyses présentées exploitent un grand nombre d'études consacrées aux expressions référentielles et anaphoriques durant les dernières décennies. Pour ne pas surcharger le texte, nous avons reporté, en bibliographie, les ouvrages de synthèse sur le fonctionnement de ces expressions en français.

3. Voir Storme et Storme ci-après.

4. Voir Fuchs, Le Goffic et Maillard 1.1. pour le découpage du texte en trois parties, établi à partir d'un examen de l'intrigue et des temps verbaux.

5. Ne sont pas pris en compte dans ce calcul les sujets qui ont la forme d’un pronom relatif ( $\$ 6: l a$ pérennité de l'espace vert $\mathrm{i}_{\mathrm{i}}$ qui $\mathrm{f}_{\mathrm{i}}$ formait un gazon subsidiaire aux pieds de Sylvie).

6. Ne sont pas pris en compte dans ce calcul les sujets de propositions participiales apposées ( $\$ 6$ : Celui-ci $i_{\mathrm{i}}$ demeura seul, dressé comme un phare au bord du canal).

\section{RÉSUMÉS}

Cet article porte sur la référence aux personnages et aux lieux dans L'Occupation des sols. Les deux types de référents sont pris en compte dans la première partie qui est consacrée aux deux premiers paragraphes du récit où sont introduits les principaux protagonistes et les lieux où ils évoluent. Les deuxième et troisième parties sont dévolues respectivement aux personnages et aux zones de l'espace urbain avec les constructions qui les occupent. Les analyses s'attachent à suivre la façon très subtile dont Jean Echenoz déplace la référence au fil du texte. Quoique ontologiquement disjoints, les personnes et les lieux se rejoignent symboliquement au travers du personnage de la mère qui est à la fois présente en tant que personne et qu'effigie, et de l'immeuble en construction qui la recouvre progressivement. Le suivi de ces évolutions par les lecteurs implique des ajustements référentiels qui sont décortiqués au fil du texte.

This paper concerns the reference to characters and places in "L'Occupation des sols". These two types of referent are taken into account in the first part of the article which is devoted to the first two paragraphs of the text wherein are introduced the major characters and the places 
where they live. The second and third parts of the paper respectively deal with characters and areas of urban space with the buildings occupying them. We examine the subtle way in which Jean Echenoz moves the reference throughout the text. Although ontologically disjoint, characters and places come together symbolically through the character of the mother who is both present as a person and as a painting representation, and through the building under construction that gradually recovers her. Monitoring these developments by readers involves referential adjustments which are tracked over the text.

\section{INDEX}

Mots-clés : expressions référentielles, anaphores, chaînes de référence, compréhension en lecture

Keywords : referential expressions, anaphora, referential chaines, reading comprehension

\section{AUTEURS}

\section{MICHEL CHAROLLES}

Professeur émérite, université Paris 3, laboratoire Lattice CNRS-ENS-Université Paris 3.

Parmi les publications :

C. Schnedecker, G. Kleiber, M. Charolles et J. David (éd.), L'Anaphore associative, Paris, Klincksieck, 1994.

M. Charolles et A. Reboul (éd.), Anaphore et Référence. Verbum, XIX, 1-2, 1997.

M. Charolles et G. Kleiber (éd.), Associative Anaphora, Journal of Pragmatics, 31, 1999.

La Référence et les expressions référentielles en français, Paris, Ophrys, 2002.

M. Charolles et M.-P. Péry-Woodley (éd.), Les Adverbiaux cadratifs, Langue française, 148, 2005.

M. Charolles, « Annotation des expressions référentielles et profondeur de traitement ", in M.-J. Béguelin et M. Fossard (éd.), Nouvelles perspectives sur l'anaphore, Bern, Peter Lang, 2014, p. 55-98.

\section{BENJAMIN STORME}

Massachusetts Institute of Technology.

Parmi les publications :

«Sicilia amissa : syntagme nominal ou proposition subordonnée? ", Revue de philologie, de littérature et d'histoire anciennes, 84 (1), 2010. 